\title{
ABSOLUTE PROPERTIES OF THE ECLIPSING BINARY STAR HY VIRGINIS
}

\author{
Claud H. Sandberg LaCY ${ }^{1,3}$ And Francis C. FeKel ${ }^{2,3,4}$ \\ ${ }^{1}$ Physics Department, University of Arkansas, Fayetteville, AR 72701, USA; clacy@ uark.edu \\ ${ }^{2}$ Center of Excellence in Information Systems, Tennessee State University, Nashville, TN 37209, USA; fekel@evans.tsuniv.edu \\ Received 2011 August 16; accepted 2011 September 27; published 2011 October 31
}

\begin{abstract}
HY Vir is found to be a double-lined F0m + F5 binary star with relatively shallow (0.3 mag) partial eclipses. Previous studies of the system are improved with 7509 differential photometric observations from the URSA WebScope and 8862 from the NFO WebScope, and 68 high-resolution spectroscopic observations from the Tennessee State University $2 \mathrm{~m}$ automatic spectroscopic telescope, and the $1 \mathrm{~m}$ coudé-feed spectrometer at Kitt Peak National Observatory. Very accurate (better than $0.5 \%$ ) masses and radii are determined from analysis of the new light curves and radial velocity curves. Theoretical models match the absolute properties of the stars at an age of about $1.35 \mathrm{~Gy}$.
\end{abstract}

Key words: binaries: eclipsing - binaries: spectroscopic - stars: fundamental parameters - stars: individual (HY Vir)

Online-only material: machine-readable and VO tables

\section{INTRODUCTION}

Eclipsing binary stars provide critical information that can be used to test our current theories of stellar evolution. By measuring accurately the changes in brightness over time (the light curve), times of minimum light (the ephemeris curve), and the pattern of changing radial velocities of the components (the radial velocity curve), orbital parameters may be determined including the masses, radii, and luminosities. These observationally determined values may then be compared with theoretical results from the current theory of stellar evolution to gauge the degree of completeness of the theory. That is the goal of this type of investigation.

The detached main-sequence eclipsing binary star HY Vir (HD 114125, HIP 64120, TYC 4960-976-1) is a relatively bright star, originally classified as F2 but now known to be F0m + F5. It was first suspected of being an eclipsing binary star by Rodriguez et al. (1988), then confirmed by Casas \& GomezForrellad (1989), who found preliminary photometric elements. These elements were improved by analyzing new photometry by Garcia-Melendo et al. (1995). Finally, near-infrared light curves were obtained by Arevalo et al. (2002) in the $J, H$, and $K$ filters and photometric elements were obtained. In all of these previous investigations, a spectroscopic mass ratio was not available, so the resulting basic parameters were poorly determined. This present study is based on the analysis of a large number (68) of new high-resolution spectrograms, combined with a very large number $(7509+8862)$ of new differential magnitudes obtained by robotic telescopes. These new results are more definitive than in previous studies and are accurate to better than $0.5 \%$. The new spectroscopic study is discussed in Section 2, the photometric study in Section 3, and the absolute properties and comparison to theory in Section 4.

\footnotetext{
3 Visiting Astronomer, Kitt Peak National Observatory, National Optical Astronomy Observatories, operated by the Association of Universities for Research in Astronomy, Inc. under a cooperative agreement with the National Science Foundation.

4 The research at Tennessee State University was supported in part by NASA, NSF, Tennessee State University, and the State of Tennessee through its Centers of Excellence program.
}

\section{SPECTROSCOPY STUDY}

\subsection{Spectroscopic Observations and Reductions}

At Fairborn Observatory from 2010 February through 2011 June we acquired 64 double-lined spectra with the Tennessee State University $2 \mathrm{~m}$ automatic spectroscopic telescope (AST), a fiber-fed echelle spectrograph, and a $2048 \times 4096$ SITeST-002A CCD. The echelle spectrograms have 21 orders that cover the wavelength range 4920-7100 $\mathrm{A}$. The resolution depended on the fiber used, and was either 0.2 or $0.3 \AA$, which produced typical signal-to-noise ratios of 35 and 70 , respectively.

In 2010 April and June, we obtained four additional doublelined spectra with the Kitt Peak National Observatory (KPNO) coudé-feed telescope, coudé spectrograph, and a TI CCD. Those spectra are centered at $6430 \AA$, cover a wavelength range of $84 \AA$, and have a resolution of $0.21 \AA$. The spectra have a signal-tonoise ratio of $\sim 150$. A portion of one of those spectra is shown in Figure 1.

Fekel et al. (2009) have provided an extensive general description of the velocity measurement for the Fairborn Observatory echelle spectra. The line profiles of HY Vir were initially compared to Gaussian functions, which, because of the rotational broadening of the stellar lines, did not match the observed profiles particularly well. Therefore, we also chose to fit the lines with empirical profiles that were rotationally broadened by shifting and adding together a set of similar profiles after weighting them according to the limb darkening adopted for the Sun. Although the rotationally broadened profiles substantially improved the fits to the lines, the measured radial velocity differences were small and the resulting changes in the orbital elements from the two different types of line fits were just $2 \sigma$ or less. Thus, although the solar limb darkening relation is not correct for the late-A/early-F primary star, using a more appropriate limb darkening relation for that component would not substantially enhance the precision of the results.

We note that we used a line list for solar-type stars, and that the resulting Fairborn velocities are on an absolute scale. Our unpublished measurements of several IAU solar-type velocity standards indicate that the Fairborn velocities have a small zeropoint offset of $-0.3 \mathrm{~km} \mathrm{~s}^{-1}$ relative to the velocities of Scarfe 


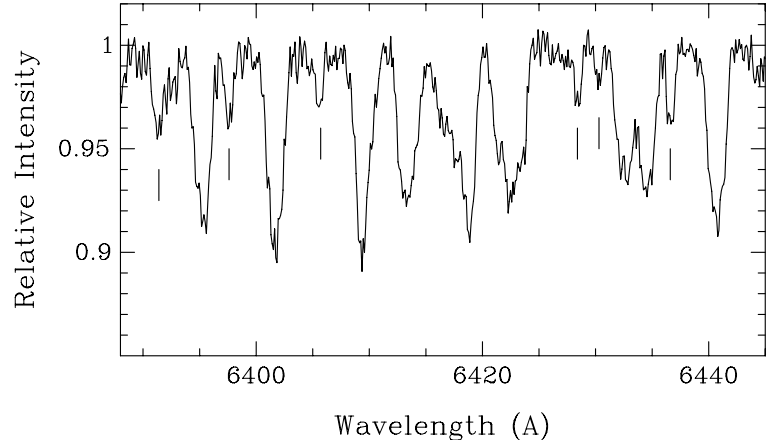

Figure 1. Portion of the spectrum of HY Vir in the red. Lines of the blueshifted secondary component are marked.

(2010). Thus, we have added $0.3 \mathrm{~km} \mathrm{~s}^{-1}$ to each of our velocities, measured with the rotational broadening function, and list them in Table 1.

Tomkin \& Fekel (2006) have discussed the measurement of the KPNO coude spectra. The KPNO velocities are relative velocities that have been determined by cross-correlation with the IAU radial velocity standard star HR 5694. From Scarfe (2010) we adopt a velocity of $54.4 \mathrm{~km} \mathrm{~s}^{-1}$ for that standard. The resulting RV measurements are listed in Table 1.

\subsection{Spectroscopic Orbit}

Circular orbits for HY Vir were determined with the algorithms SB1C and SB2C (D. Barlow 1998, private communication), which use differential corrections to compute improved orbital elements. With 64 observations from Fairborn Observatory and 4 from KPNO we initially computed separate orbits for components A and B with the orbital period fixed at the photometrically determined value. From the variances of the two solutions, the velocities of $\mathrm{A}$ and $\mathrm{B}$ have been given weights of 1.0 and 0.3 , respectively. Because we obtained only four observations from KPNO, component velocities from those spectra have been assigned the same weights as those of the much more numerous Fairborn velocities. The center-of-mass velocities for the two components differ by $0.5 \mathrm{~km} \mathrm{~s}^{-1}$, a $2 \sigma$ result, and so we combined the appropriately weighted velocities into a doublelined circular orbit solution and list the resulting orbital elements and related parameters in Table 2. We also computed a doublelined solution with the period as a free parameter. The result was $P=2.7323353 \pm 0.0000112$ days, a value in excellent accord with the much more accurate photometrically determined value of $2.7323344 \pm 0.0000010$ days.

For a circular orbit the element $T$, a time of periastron passage, is undefined. Thus, as recommended by Batten et al. (1989), $T_{\mathrm{o}}$, a time of maximum velocity for the primary, is used instead. Thus, the zero phase of our spectroscopic orbit is 0.25 earlier than that computed from the primary eclipse ephemeris. The final fitted spectroscopic orbit is shown in Figure 2.

\subsection{Spectral Types and Rotational Velocities}

Strassmeier \& Fekel (1990) identified several luminositysensitive and temperature-sensitive line ratios in the 6430-6465 $\AA$ region. They employed those critical line ratios and the general appearance of the spectrum as spectral-type criteria. However, for stars that are hotter than early-G spectral class, the line ratios in that wavelength region have little sensitivity to luminosity. Thus, for the components of HY Vir, we have used the entire $84 \AA$ spectral region of our KPNO observations in an attempt to estimate just the spectral classes of the

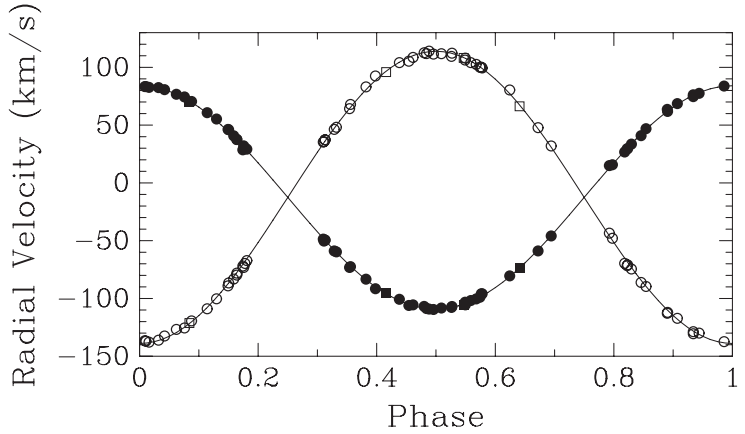

Figure 2. Radial velocities and the fitted orbits for HY Vir. Filled and open symbols represent the primary and secondary, respectively. Circles: Fairborn Observatory; squares: KPNO.

individual components. The luminosity class may be determined by computing the absolute visual magnitude with the Hipparcos parallax and comparing that magnitude to evolutionary tracks.

The best KPNO spectrum of HY Vir was compared with the spectra of a number of A- and F-type stars primarily from the lists of Abt \& Morrell (1995) and Fekel (2003). The reference-star spectra were obtained at KPNO with the same telescope, spectrograph, and detector as our binary star spectra. To facilitate a comparison, various combinations of the reference-star spectra were rotationally broadened, shifted in radial velocity, appropriately weighted, and added together with a computer program developed by Huenemoerder \& Barden (1984) and Barden (1985) in an attempt to reproduce the binary spectra.

For HY Vir that analysis has met with limited success because the primary is an Am star. Classical Am stars have spectral classes of A4-F1, determined from their hydrogen lines (Abt $\&$ Morrell 1995). Such stars are noted as having peculiar spectra because lines of their metallic elements such as iron and strontium are stronger than expected compared to the hydrogen classification, while elements such as calcium and scandium are weaker (Abt \& Morrell 1995). There are no hydrogen lines in our limited $6430 \AA$ wavelength region, and the iron and calcium abundance peculiarities vary from star to star, making it impossible to adequately characterize the combined spectrum of the two components with our limited number of reference spectra.

Although a full characterization of the two components is not possible, we have the following comments. The weaker and narrower lines of the secondary are consistent with an F5 spectral class. The Fe I lines of the primary in the $6430 \AA$ region are very strong and even adopting the spectrum of a solar-abundance F5 star for both components does not reproduce the strength of the primary's Fe I lines. Thus, iron in the primary's outer atmosphere is very overabundant, and no estimate of the luminosity ratio can be determined by comparing line equivalent widths of the two stars.

Following the procedure of Fekel (1997), from our four KPNO spectra we compute average $v \sin i$ values of $48 \pm 1$ and $24 \pm 2 \mathrm{~km} \mathrm{~s}^{-1}$ for $\mathrm{A}$ and $\mathrm{B}$, respectively, where the uncertainties are estimated. Many of the Fairborn Observatory spectra have high enough signal-to-noise ratios to enable good $v \sin i$ determinations. From those spectra we compute averages of 48 and $22 \mathrm{~km} \mathrm{~s}^{-1}$ for $\mathrm{A}$ and $\mathrm{B}$, respectively. We estimate that these values have uncertainties similar to those from the KPNO spectra although the formal uncertainties are about three times smaller than those values. 
Table 1

Radial Velocities of HY Vir

\begin{tabular}{|c|c|c|c|c|c|}
\hline HJD-2400000 & Orbital Phase & $\begin{array}{l}\text { Primary RV } \\
\left(\mathrm{km} \mathrm{s}^{-1}\right)\end{array}$ & $\begin{array}{c}\text { Primary } O-C \\
\quad\left(\mathrm{~km} \mathrm{~s}^{-1}\right)\end{array}$ & $\begin{array}{c}\text { Secondary RV } \\
\left(\mathrm{km} \mathrm{s}^{-1}\right)\end{array}$ & $\begin{array}{c}\text { Secondary } O-C \\
\left(\mathrm{~km} \mathrm{~s}^{-1}\right)\end{array}$ \\
\hline 55244.874 & 0.576 & -95.9 & 2.3 & 100.2 & 0.6 \\
\hline 55246.929 & 0.328 & -58.9 & -0.7 & 46.3 & -1.0 \\
\hline 55247.929 & 0.694 & -45.9 & -0.4 & 32.0 & 1.3 \\
\hline 55250.929 & 0.792 & 15.0 & 2.1 & -43.5 & 2.3 \\
\hline 55251.930 & 0.159 & 40.8 & 0.9 & -83.2 & -2.1 \\
\hline 55253.930 & 0.891 & 61.9 & -0.3 & -112.0 & -1.7 \\
\hline 55257.933 & 0.356 & -72.5 & -0.4 & 67.9 & 2.4 \\
\hline 55259.933 & 0.088 & 70.5 & 0.8 & -119.4 & 0.8 \\
\hline 55260.934 & 0.454 & -106.1 & -1.0 & 105.3 & -3.4 \\
\hline 55267.934 & 0.016 & 82.7 & -0.9 & -137.8 & 0.5 \\
\hline 55268.934 & 0.382 & -83.3 & 0.4 & 83.1 & 2.4 \\
\hline 55270.934 & 0.114 & 60.8 & 0.5 & -108.9 & -1.0 \\
\hline 55271.934 & 0.480 & -106.9 & 1.4 & 112.6 & -0.3 \\
\hline 55272.934 & 0.846 & 40.9 & -1.3 & -86.2 & -2.0 \\
\hline 55274.934 & 0.578 & -95.9 & 1.8 & 99.5 & 0.4 \\
\hline 55275.934 & 0.944 & 77.5 & -0.6 & -130.0 & 1.2 \\
\hline 55276.934 & 0.310 & -48.6 & -0.6 & 35.4 & 1.4 \\
\hline 55282.886 & 0.488 & -109.2 & -0.4 & 114.0 & 0.4 \\
\hline 55283.886 & 0.854 & 46.9 & 0.6 & -89.6 & -0.1 \\
\hline 55285.836 & 0.568 & -100.4 & 0.0 & 102.6 & 0.0 \\
\hline 55286.836 & 0.934 & 76.3 & 0.5 & -130.5 & -2.3 \\
\hline 55289.836 & 0.032 & 82.5 & 0.4 & -136.1 & 0.3 \\
\hline 55290.836 & 0.398 & -91.6 & -1.7 & 92.5 & 3.7 \\
\hline 55292.836 & 0.130 & 55.3 & 1.6 & -100.3 & -1.1 \\
\hline 55293.836 & 0.496 & -109.5 & -0.5 & 111.5 & -2.4 \\
\hline 55296.651 & 0.526 & -107.8 & 0.0 & 109.5 & -2.7 \\
\hline 55297.646 & 0.890 & 63.6 & 1.6 & -112.8 & -2.7 \\
\hline 55300.640 & 0.986 & 83.8 & 0.1 & -137.4 & 1.1 \\
\hline 55301.646 & 0.354 & -73.2 & -1.9 & 64.3 & -0.2 \\
\hline 55303.890 & 0.175 & 32.1 & 1.0 & -73.2 & -3.6 \\
\hline 55307.644 & 0.549 & -105.5 & -1.0 & 108.0 & 0.1 \\
\hline 55311.777 & 0.062 & 76.7 & -0.1 & -126.8 & 2.7 \\
\hline $55311.837^{\mathrm{a}}$ & 0.084 & 70.0 & -0.9 & -121.3 & 0.5 \\
\hline 55312.744 & 0.416 & -95.4 & 0.5 & 95.9 & -0.8 \\
\hline $55312.805^{\mathrm{a}}$ & 0.438 & -100.7 & 1.2 & 104.1 & -0.4 \\
\hline 55313.787 & 0.798 & 15.7 & -0.3 & -47.9 & 1.9 \\
\hline $55314.816^{\mathrm{b}}$ & 0.174 & 28.6 & -3.1 & -71.9 & -1.4 \\
\hline 55318.778 & 0.624 & -80.5 & 0.6 & 80.4 & 3.1 \\
\hline 55319.829 & 0.009 & 83.4 & -0.5 & -136.6 & 2.2 \\
\hline 55323.797 & 0.461 & -105.5 & 0.7 & 108.6 & -1.6 \\
\hline 55325.755 & 0.178 & 30.2 & 0.4 & -69.7 & -1.8 \\
\hline 55326.770 & 0.549 & -103.2 & 1.3 & 106.3 & -1.6 \\
\hline 55327.748 & 0.907 & 68.6 & 0.5 & -117.2 & 0.9 \\
\hline 55337.725 & 0.559 & -101.8 & 0.8 & 104.1 & -1.3 \\
\hline 55356.716 & 0.509 & -108.3 & 0.6 & 111.8 & -1.9 \\
\hline 55363.729 & 0.076 & 74.4 & 1.1 & -125.5 & -0.6 \\
\hline 55366.702 & 0.164 & 37.5 & 0.3 & -79.8 & -2.1 \\
\hline $55367.748^{\mathrm{a}}$ & 0.547 & -105.6 & -0.6 & 108.2 & -0.3 \\
\hline $55370.738^{\mathrm{a}}$ & 0.641 & -73.8 & -0.2 & 66.3 & -1.2 \\
\hline 55376.718 & 0.830 & 33.6 & -0.2 & -74.5 & -1.4 \\
\hline 55516.036 & 0.818 & 26.7 & -0.9 & -69.6 & -4.5 \\
\hline 55572.029 & 0.311 & -50.3 & -1.7 & 35.7 & 1.0 \\
\hline 55578.891 & 0.822 & 29.5 & -0.4 & -71.6 & -3.6 \\
\hline 55587.984 & 0.150 & 46.0 & 1.9 & -86.6 & 0.0 \\
\hline 55598.997 & 0.181 & 29.2 & 1.1 & -67.2 & -1.5 \\
\hline 55624.930 & 0.672 & -58.9 & -1.0 & 47.8 & 0.8 \\
\hline 55629.998 & 0.527 & -107.0 & 0.7 & 112.3 & 0.2 \\
\hline 55639.005 & 0.823 & 29.7 & -0.7 & -71.0 & -2.3 \\
\hline 55647.800 & 0.042 & 80.7 & 0.0 & -132.5 & 2.0 \\
\hline 55655.701 & 0.934 & 74.7 & -1.1 & -128.7 & -0.5 \\
\hline 55667.666 & 0.313 & -49.4 & 0.3 & 37.3 & 1.1 \\
\hline 55681.795 & 0.484 & -108.7 & -0.1 & 111.9 & -1.4 \\
\hline 55691.810 & 0.149 & 46.3 & 1.7 & -89.1 & -1.8 \\
\hline 55702.780 & 0.164 & 37.7 & 0.6 & -78.1 & -0.7 \\
\hline
\end{tabular}


Table 1

(Continued)

\begin{tabular}{lccccc}
\hline \hline HJD-2400000 & Orbital Phase & $\begin{array}{c}\text { Primary RV } \\
\left(\mathrm{km} \mathrm{s}^{-1}\right)\end{array}$ & $\begin{array}{c}\text { Primary } O-C \\
\left(\mathrm{~km} \mathrm{~s}^{-1}\right)\end{array}$ & $\begin{array}{c}\text { Secondary RV } \\
\left(\mathrm{km} \mathrm{s}^{-1}\right)\end{array}$ & $\begin{array}{c}\text { Secondary } O-C \\
\left(\mathrm{~km} \mathrm{~s}^{-1}\right)\end{array}$ \\
\hline 55718.755 & 0.011 & 83.4 & -0.4 & -137.0 & 1.7 \\
55727.777 & 0.313 & -49.6 & 0.0 & 37.2 & 1.1 \\
55736.688 & 0.574 & -99.0 & -0.2 & 100.1 & -0.4 \\
55738.759 & 0.332 & -59.7 & 0.4 & 48.2 & -1.6 \\
\hline
\end{tabular}

Notes.

a Observation obtained at KPNO.

${ }^{\mathrm{b}}$ Primary velocity rejected from final spectroscopic solution due to $3 \sigma$ residual.

Table 2

Spectroscopic Orbital Elements and Related Parameters of HY Vir

\begin{tabular}{lc}
\hline \hline Parameter & Value \\
\hline$P^{\mathrm{a}}$ (days) & 2.7323344 (adopted) \\
$T_{\mathrm{o}}$ b $(\mathrm{HJD})$ & $2454470.0486 \pm 0.0007$ \\
$e$ & 0.0 (adopted) \\
$\omega_{\mathrm{A}}(\mathrm{deg})$ & 0.0 (fixed) \\
$K_{\mathrm{A}}\left(\mathrm{km} \mathrm{s}^{-1}\right)$ & $96.56 \pm 0.16$ \\
$K_{\mathrm{B}}\left(\mathrm{km} \mathrm{s}^{-1}\right)$ & $126.44 \pm 0.29$ \\
$\gamma\left(\mathrm{km} \mathrm{s}^{-1}\right)$ & $-12.52 \pm 0.11$ \\
$m_{\mathrm{A}} \sin ^{3} i($ solar masses $)$ & $1.7841 \pm 0.0092$ \\
$m_{\mathrm{B}} \sin ^{3} i($ solar masses $)$ & $1.3625 \pm 0.0055$ \\
$a_{\mathrm{A}} \sin i\left(10^{6} \mathrm{~km}\right)$ & $3.6279 \pm 0.0060$ \\
$a_{\mathrm{B}} \sin i\left(10^{6} \mathrm{~km}\right)$ & $4.7506 \pm 0.0110$ \\
Standard error of a unit weight observation $\left(\mathrm{km} \mathrm{s}^{-1}\right)$ & 1.0 \\
\hline
\end{tabular}

Notes.

${ }^{a}$ Photometric period.

b Time of maximum primary velocity.

Table 3

Times of Eclipse of HY Vir

\begin{tabular}{lccrc}
\hline \hline HJD-2400000 & Type & Accuracy & Residual & Ref. \\
\hline 47239.60510 & 2 & 0.00100 & -0.00341 & 1 \\
47627.60069 & 2 & 0.00030 & 0.00069 & 2 \\
49813.47041 & 2 & 0.00030 & 0.00289 & 2 \\
49817.56624 & 1 & 0.00030 & 0.00022 & 2 \\
53470.69660 & 1 & 0.00090 & -0.00051 & 3 \\
53492.55450 & 1 & 0.00180 & -0.00128 & 3 \\
54159.25270 & 1 & 0.00200 & 0.00732 & 4 \\
55280.86700 & 2 & 0.00030 & -0.00165 & 5 \\
55295.89860 & 1 & 0.00060 & 0.00211 & 5 \\
55306.82570 & 1 & 0.00030 & -0.00013 & 5 \\
55317.75360 & 1 & 0.00040 & -0.00156 & 5 \\
55332.78770 & 2 & 0.00050 & 0.00470 & 5 \\
55369.66620 & 1 & 0.00030 & -0.00332 & 5 \\
55590.98810 & 1 & 0.00030 & -0.00050 & 5 \\
55623.77670 & 1 & 0.00030 & 0.00008 & 6 \\
55716.67990 & 1 & 0.00030 & 0.00391 & 6 \\
\hline
\end{tabular}

References: (1) Rodriguez et al. 1988; (2) Garcia-Melendo et al. 1995; (3) Ogloza et al. 2008; (4) Nagai 2008; (5) Lacy 2011; (6) this paper.

\section{PHOTOMETRIC STUDY}

\subsection{Times of Minimum and the Orbital Period}

All published times of minimum (see Table 3) were gathered and fitted with a linear ephemeris equation:

$\operatorname{Min} I(\mathrm{HJD})=2,454,470.7315(7)+2.7323344(10) \times E$.

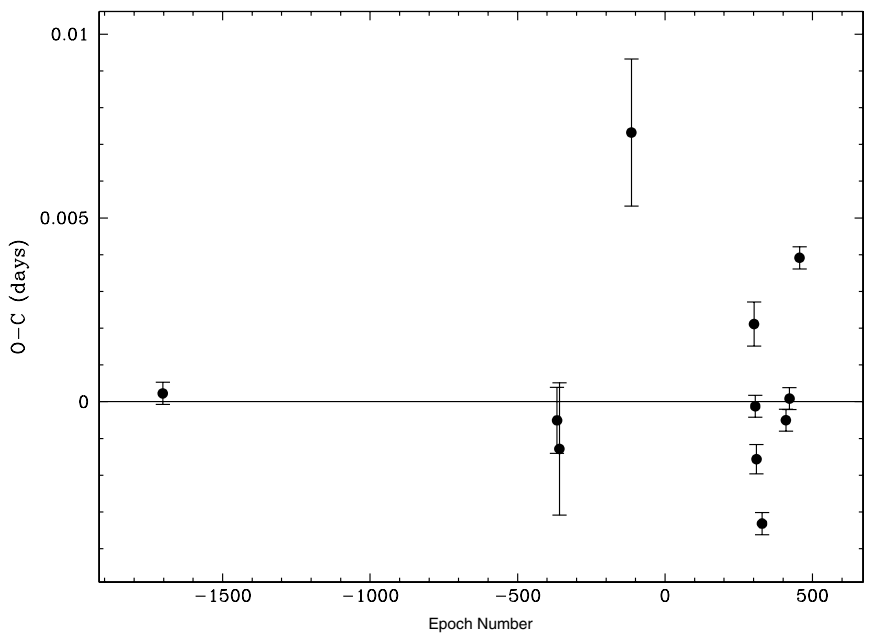

Figure 3. Deviations of dates of primary minimum from the adopted linear ephemeris. The residuals scatter randomly around zero.

In this process, the reduced chi-squared was adjusted to unity by scaling the observational uncertainties by a constant, so the error estimates of the fitted period and zero epoch are realistic. The fit is shown in Figure 3.

\subsection{Interstellar Reddening and Mean Temperature}

Color indices in the $u v b y \beta$ photometric system have been published by Hauck \& Mermilliod (1998) based on two measurements. This photometric system is designed to allow accurate estimation of the interstellar reddening. The $\beta$ value places the mean spectral type in the A-F star border region. The $u v b y \beta$ indices indicate that the primary is an F0m star, and Renson \& Manfroid (2009) list it as F2 Sr Eu Cr based on spectra. Following the precepts of Crawford \& Barnes (1974) but using the standard relations of Perry \& Johnson (1982), we find an interstellar reddening value of $E(b-y)=0.019 \mathrm{mag}$ and a visual absorption of $\mathrm{Av}=0.082 \mathrm{mag}$. The value of $\delta m_{1}$, through the calibration of Stromgren (1966), gives a very high surface value of $[\mathrm{Fe} / \mathrm{H}]=1.0 \pm 0.1$, confirming the metallic-lined character of the spectrum. The temperature calibration of Popper (1980), which is based primarily on that of Hayes (1978), gives a mean value of $6760 \pm 130 \mathrm{~K}(\mathrm{~F} 2 \mathrm{~m})$ based on the value of $(b-y)_{\mathrm{o}}$, where we have doubled the formal uncertainty because of the unusual surface chemistry of the stars. We have explored the use of other temperature scales, such as those of Alonso et al. (1996) and Casagrande et al. (2010), but our star's metallicity is beyond the range of the calibration data for those scales. Thus, we prefer the scale of Popper (1980), but recognize that the formal error is probably too small for this very metallic star. 
Table 4

URSA Differential Photometry of HY Vir

\begin{tabular}{lcc}
\hline \hline Orbital Phase & $\Delta V$ & HJD-2400000 \\
\hline 0.08295 & -0.602 & 54943.65193 \\
0.08318 & -0.624 & 54943.65257 \\
0.08340 & -0.633 & 54943.65317 \\
0.08363 & -0.627 & 54943.65378 \\
0.08385 & -0.643 & 54943.65438
\end{tabular}

(This table is available in its entirety in machine-readable and Virtual Observatory (VO) forms in the online journal. A portion is shown here for guidance regarding its form and content.)

Table 5

NFO Differential Photometry of HY Vir

\begin{tabular}{lcc}
\hline \hline Orbital Phase & $\Delta V$ & HJD-2400000 \\
\hline 0.01080 & -0.403 & 54940.72247 \\
0.01113 & -0.401 & 54940.72337 \\
0.01145 & -0.402 & 54940.72424 \\
0.01179 & -0.405 & 54940.72516 \\
0.01210 & -0.401 & 54940.72602 \\
\hline
\end{tabular}

(This table is available in its entirety in machine-readable and Virtual Observatory (VO) forms in the online journal. A portion is shown here for guidance regarding its form and content.)

\subsection{Differential Photometry}

One of the telescopes used to obtain the differential photometry is the URSA WebScope, which consists of a Meade $10 \mathrm{inch}$ f/6.3 LX-200 telescope with a Santa Barbara Instruments Group ST8 CCD camera (binned $2 \times 2$ to produce $765 \times 510$ pixel images with $2.3 \operatorname{arcsec}^{2}$ pixels) inside a Technical Innovations Robo-Dome, and controlled automatically by an Apple Macintosh G4 computer. The observatory is located on top of Kimpel Hall on the Fayetteville campus, with the control room directly beneath the observatory inside the building. Ten-second exposures through a Bessel $V$ filter $(2.0 \mathrm{~mm}$ of GG 495 and $3.0 \mathrm{~mm}$ of BG 39) were read out and downloaded to the control computer over a $30 \mathrm{~s}$ interval, then the next exposure was begun. The observing cadence was therefore about 40 s per observation. The variable star would frequently be monitored continuously for 2-4 hr. HY Vir was observed by URSA on 56 nights during parts of three observing seasons from 2009 April 22 to 2011 July 2, yielding 7509 observations.

The other telescope we used is the NFO WebScope, a refurbished 24 inch Group 128 cassegrain reflector with a $2 \mathrm{~K} \times$ 2K Kodak CCD camera, located near Silver City, NM (Grauer et al. 2008). Observations consisted of $10 \mathrm{~s}$ exposures through a Bessel $V$ filter. HY Vir was observed by NFO on 135 nights during parts of three observing seasons from 2009 April 19 to 2011 June 21, yielding 8862 observations.

The images were analyzed by a virtual measuring engine application written by Lacy that flat-fielded the URSA images (the NFO images are flat-fielded before distribution); automatically located the variable, comparison, and check stars in the image; measured their brightnesses; subtracted the corresponding sky brightness; and corrected for the differences in airmass between the stars. Extinction coefficients were determined nightly from the comparison star measurements. They averaged 0.25 mag airmass $^{-1}$ at URSA (they ranged from 0.20 to 0.30 mag airmass $^{-1}$ ), 0.18 mag airmass $^{-1}$ at the NFO (they ranged from 0.12 to 0.25 mag airmass $^{-1}$ ). The comparison stars

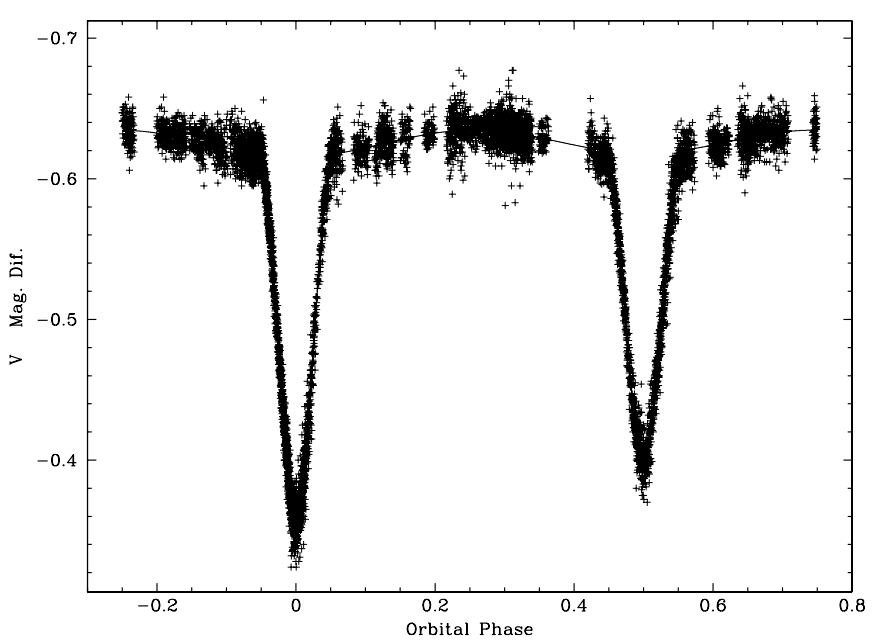

Figure 4. Light curve of HY Vir from the URSA WebScope.

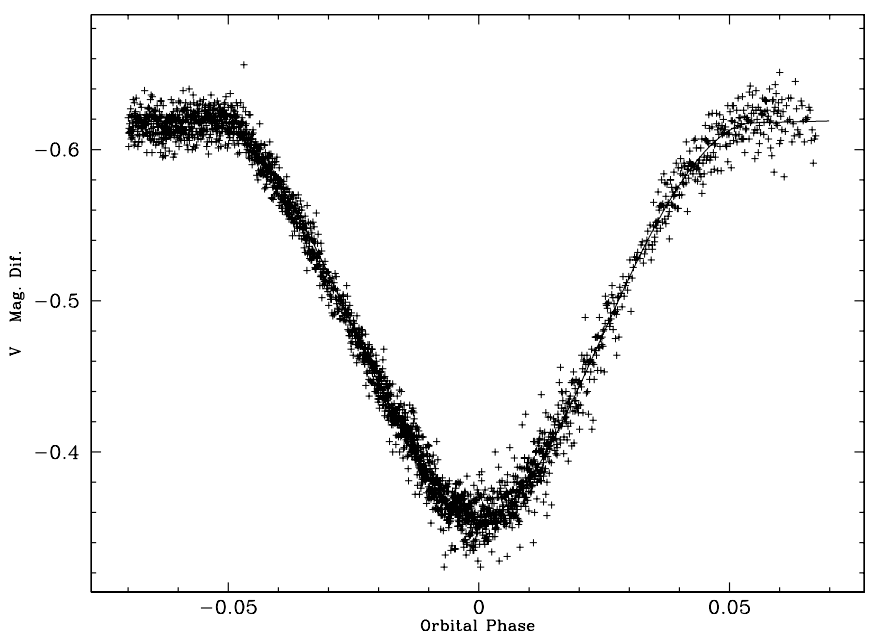

Figure 5. URSA observations of the primary eclipse of HY Vir.

were TYC 4960-0015-1 ( $V=8.71$, F0) and TYC 4960-0320-1 $(V=10.11, \mathrm{~F} 8)$. Both comparison stars are within 7 arcmin of the variable star. The comparison star magnitude differences were constant at the level of $0.016 \mathrm{mag}$ (URSA) and $0.015 \mathrm{mag}$ (NFO) for the standard deviation of the magnitude differences, and $0.012 \mathrm{mag}$ (URSA) and $0.016 \mathrm{mag}$ (NFO) for the standard deviation of the nightly means. These values are larger than usual because of the relative faintness of the fainter comparison star. The sum of the fluxes of both comparison stars was converted to a magnitude called "comparisons". The resulting 7509 (URSA) and 8862 (NFO) $V$ magnitude differences (variablecomparisons) are listed in Tables 4 and 5 (without any nightly corrections) and are shown in Figures 4-9 (after the nightly corrections discussed below have been added).

We noticed early on during the observations that the NFO magnitudes showed a small but significant offset from night to night, on the order of a hundredth of a magnitude. The origin of the offset is a variation in responsivity across the field of view of the NFO combined with imprecise centering from night to night. These variations are a well-known effect of the optics when using wide-field imaging telescopes such as the NFO. We have removed most of this variation by using dithered exposures of open star clusters to measure this variation, fitting a twodimensional polynomial (see Selman 2004) and removing the variation during initial reductions (photometric flat). The URSA 


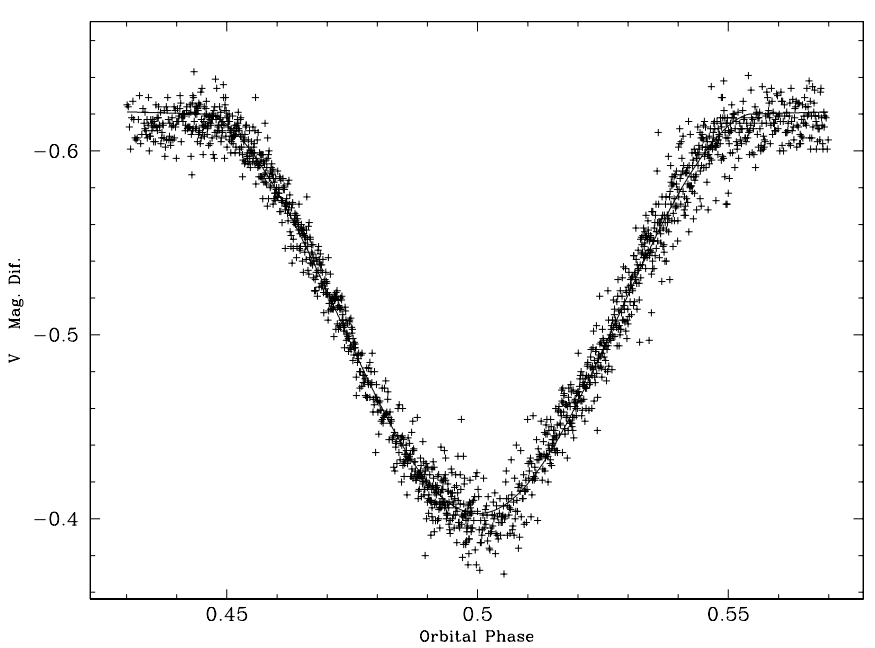

Figure 6. URSA observations of the secondary eclipse of HY Vir.

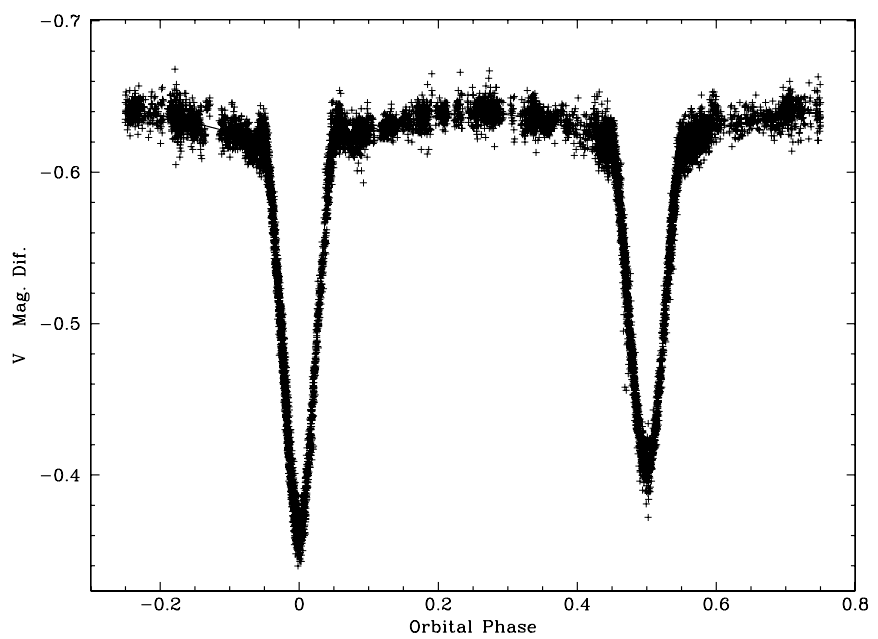

Figure 7. NFO light curve of HY Vir.

observations, moreover, show this kind of effect to a very much smaller extent.

\subsection{Photometric Orbit}

The light curve fitting was done with the NDE model as implemented in the code jktebop (Etzel 1981; Popper \& Etzel 1981, Southworth et al. 2007), and the ephemeris adopted is that of Section 3. The main adjustable parameters are the relative central surface brightness of the secondary star $\left(J_{\mathrm{B}}\right)$ in units of that of the primary, the sum of relative radius of the primary and secondary $\left(r_{\mathrm{A}}+r_{\mathrm{B}}\right)$ in units of the separation, the ratio of radii $\left(k=r_{\mathrm{B}} / r_{\mathrm{A}}\right)$, the inclination of the orbit $(i)$, and the geometric factors $e \cos w$ and $e \sin w$ which account for the orbital eccentricity. Auxiliary parameters needed in the analysis include the gravity-brightening exponent, which we adopt as 0.18 for both the primary and 0.24 for the secondary based on their temperatures (Claret 1998). Limb-darkening coefficients (u) were adopted as the average of five recent theoretical values (Van Hamme 1993; Diaz-Cordoves et al. 1995; Claret 2000; Claret \& Hauschildt 2003). The mass ratio $\left(q=M_{\mathrm{B}} / M_{\mathrm{A}}=\right.$ 0.763 ) was adopted from the spectroscopic analysis in Section 2. Other adjusted parameters were the magnitude at quadrature and the phase of primary eclipse. The amount of "reflected light" was calculated from bolometric theory (see Popper \& Etzel 1981).

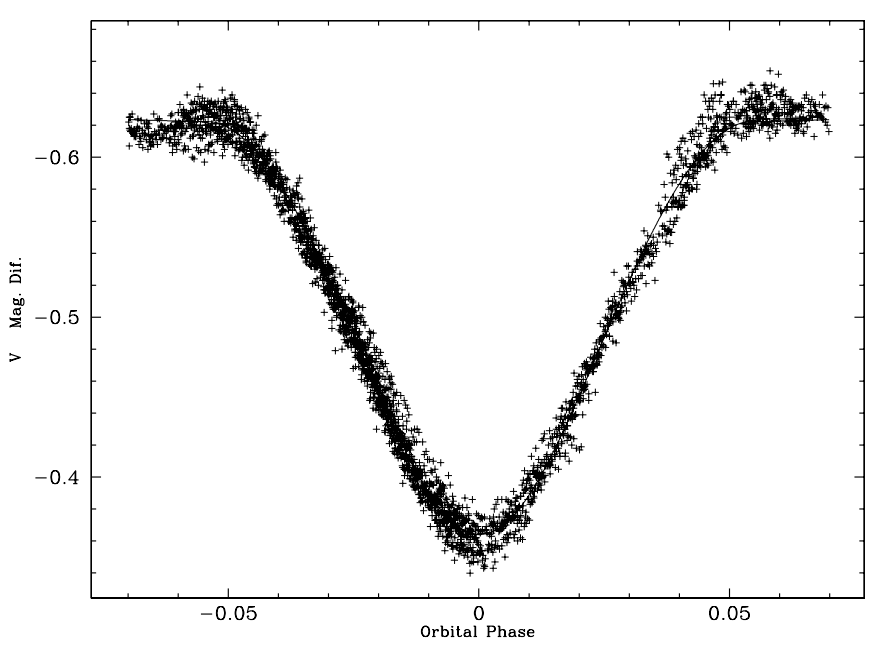

Figure 8. NFO observations of the primary eclipse of HY Vir.

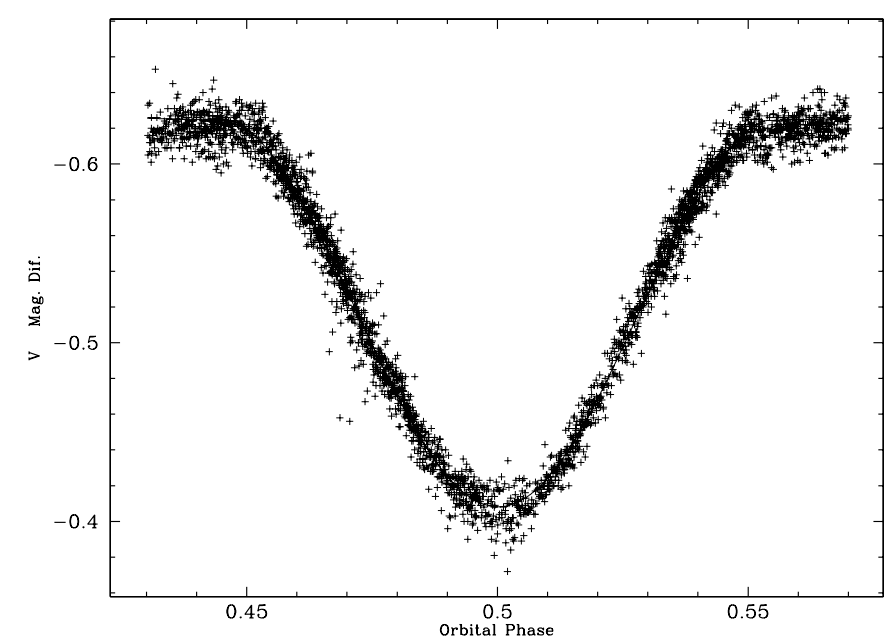

Figure 9. NFO observations of the secondary eclipse of HY Vir.

The fitting procedure converged to a solution for both the URSA and NFO data sets (Table 6).

Examination of the residuals showed that small but significant night-to-night residual variations remained in the NFO data even after application of the photometric flat, and to a much smaller extent, were also present in the URSA data. The fact that they are essentially absent from the URSA data, which were obtained contemporaneously with the NFO data, shows that they are not intrinsic variations in the stars' brightnesses, but are only optical effects due to the type of telescope used. We have applied nightly corrections based on the initial photometric orbits to the data sets to remove these observational effects. The number of nights on which these adjustments were made is listed in Table 6 as "Corrections." Fits to the "corrected" data then show significantly reduced residual variance, and we have adopted these improved fits (Table 7) for further use.

Lacy et al. (1987) showed that the difference in visual surface brightness parameter, $\Delta F_{\mathrm{v}}$, is related to the normalized $V$-band central surface brightness of the secondary star in eclipsing binaries: $\Delta F_{\mathrm{v}}=0.25 \log J_{\mathrm{c}}{ }^{\prime}$. Here, $J_{\mathrm{c}}$ is a parameter that is fitted in the jktebop code that we use to model the light curves. Popper's (1980) Table 1 gives the relationship between the visual surface brightness parameter $F_{\mathrm{v}}$ and the stellar temperature, thus the difference in temperature is readily and very accurately determined from the $V$-filter light curve fit alone. It is not 
Table 6

Photometric Orbital Parameters for HY Vir in the $V$ band

\begin{tabular}{lccc}
\hline \hline Parameter & URSA & NFO & Adopted \\
\hline$J_{\mathrm{B}} / J_{\mathrm{A}}$ & $0.8226 \pm 0.0021$ & $0.8319 \pm 0.0016$ & $0.824 \pm 0.005$ \\
$r_{\mathrm{A}}=R_{\mathrm{A}} / a$ & $0.2310 \pm 0.0006$ & $0.2303 \pm 0.0011$ & $0.2306 \pm 0.006$ \\
$r_{\mathrm{B}} /=R_{\mathrm{B}} / a$ & $0.1259 \pm 0.0006$ & $0.1240 \pm 0.0004$ & $0.1248 \pm 0.0006$ \\
$k=r_{\mathrm{B}} / r_{\mathrm{A}}$ & $0.545 \pm 0.009$ & $0.539 \pm 0.006$ & $0.541 \pm 0.004$ \\
$i(\mathrm{deg})$ & $81.59 \pm 0.15$ & $81.64 \pm 0.11$ & $81.62 \pm 0.10$ \\
$u_{\mathrm{A}}$ & $0.591 \pm 0.023$ fixed & $0.591 \pm 0.023$ fixed & $0.591 \pm 0.023 \mathrm{fixed}$ \\
$u_{\mathrm{B}}$ & $0.603 \pm 0.024$ fixed & $0.603 \pm 0.024$ fixed & $0.603 \pm 0.024$ fixed \\
$y_{\mathrm{A}}$ & $0.18 \pm 0.03$ fixed & $0.18 \pm 0.03$ fixed & $0.18 \pm 0.03$ fixed \\
$y_{\mathrm{B}}$ & $0.24 \pm 0.03$ fixed & $0.24 \pm 0.03$ fixed & $0.24 \pm 0.03$ fixed \\
$q=m_{\mathrm{B}} / m_{\mathrm{A}}$ & $0.763 \pm 0.04$ fixed & $0.763 \pm 0.04$ fixed & $0.763 \pm 0.04$ fixed \\
$L_{\mathrm{A}} /\left(L_{\mathrm{A}}+L_{\mathrm{B}}\right)$ & $0.8057 \pm 0.0015$ & $0.8078 \pm 0.0011$ & $0.8068 \pm 0.0010$ \\
$\sigma$ (mmag) & 10.3119 & 9.0343 & \\
$N$ & 7509 & 8862 & 135 \\
Corrections & 56 & &
\end{tabular}

Table 7

Absolute Properties of HY Vir

\begin{tabular}{lcc}
\hline \hline Parameter & Primary & Secondary \\
\hline Mass (solar masses) & $1.838 \pm 0.009$ & $1.404 \pm 0.006$ \\
Radius (solar radii) & $2.806 \pm 0.008$ & $1.519 \pm 0.008$ \\
$\log g\left(\mathrm{~cm} \mathrm{~s}^{-2}\right)$ & $3.806 \pm 0.003$ & $4.222 \pm 0.004$ \\
Eccentricity & $48 \pm 1$ & $23 \pm 2$ \\
$v$ sin $i\left(\mathrm{~km} \mathrm{~s}^{-1}\right)$ (observed value) & $52.0 \pm 0.2$ & $28.1 \pm 0.1$ \\
$v_{\text {sync }}\left(\mathrm{km} \mathrm{s}^{-1}\right)$ (equatorial) & \multicolumn{2}{c}{$12.176 \pm 0.025$} \\
Semi-major axis (solar radii) & $3.836 \pm 0.008$ & $3.816 \pm 0.008$ \\
$\log T_{\text {eff }}$ & $1.20 \pm 0.04$ & $0.58 \pm 0.04$ \\
$\log L$ (solar units) & $1.66 \pm 0.08$ & $3.21 \pm 0.08$ \\
$M_{\mathrm{V}}$ (mag) & $3.835 \pm 0.008$ & $3.814 \pm 0.008$ \\
$F_{\mathrm{V}}$ & \multicolumn{2}{c}{$0.019 \pm 0.008$} \\
$E_{\mathrm{b}-\mathrm{y} \text { reddening (mag) }}$ & \multicolumn{2}{c}{$6.30 \pm 0.20$} \\
$m-M$ (mag) & \multicolumn{2}{c}{$182 \pm 18$} \\
Distance (pc) & \multicolumn{2}{c}{} \\
\hline
\end{tabular}

necessary to have additional light curves in different bandpasses in order to determine accurately the temperature difference if this method is used.

Tests for third light showed that it is not present at significant levels (it was not significantly different from zero when allowed to vary), and tests for eccentricity showed that it is not significantly different from zero.

We have explored the use of nonlinear limb-darkening laws (quadratic, logarithmic, and square-root), with theoretical values of limb-darkening parameters interpolated for the temperatures and $\log g$ values of our stars, based on the theoretical works of Van Hamme (1993), Diaz-Cordoves et al. (1995), Claret (2000), and Claret \& Hauschildt (2003). As a result of a dozen such trials, only the logarithmic law of Claret \& Hauschildt (2003) showed a tiny improvement (less than $0.1 \%$ in the residual error). We do not consider this to be a significant improvement over the linear law. This confirms the tests carried out by Lacy et al. (2008), concluding that the model fits to a few thousand data points of our precision are not be improved significantly by the inclusion of nonlinear limb-darkening coefficients.

A number of tests have been made comparing the NDE model used by jktebop with more complicated models (Popper \& Etzel 1981; North \& Zahn 2004) including the WD model. The primary results of these studies are that the limits for high-accuracy determination of parameters such as the radii, inclination, etc., with the NDE model are component oblateness less than 0.04 and mean radii less than 0.25 . Since the HY Vir properties are all within these limits, we do not feel the need to use a more complicated model in this case.

\section{ABSOLUTE PROPERTIES AND COMPARISON WITH THEORY}

The combination of the spectroscopic results of Table 2 with the light curve results in Table 6 leads to the absolute dimensions and masses for HY Vir shown in Table 7. Table 1 of Popper (1980) has been used for the radiative quantities. He adopted the bolometric corrections of Hayes (1978). The masses are determined to an accuracy of $0.5 \%$ (standard error), and the radii are good to about $0.5 \%$ (standard error). We have estimated the uncertainties in the effective temperatures to be $130 \mathrm{~K}$ (standard error) to account for possible systematic errors in the photometry and in the calibrations of Popper (1980). The theoretical equatorial rotational velocities corresponding to the mean orbital motion (circular rate) are probably not significantly different from the observed $v \sin i$ values.

The distance we derive for the system corresponds to a parallax of $p=5.50 \pm 0.54$ mas. The Hipparcos catalogue lists the parallax as $6.13 \pm 0.87$ mas, not significantly different from our result. The revised Hipparcos parallax (van Leeuwen 2007 , p. 350) of $5.99 \pm 0.63$ mas improved the agreement.

The accurate mass, radius, temperature, and $v$ sin $i$ determinations for HY Vir are compared in this section with theoretical models of stellar evolution. If we equate the orbital inclination and the rotational inclination, the measured $v \sin i$ values from Table 7 are increased by $0.5 \mathrm{~km} \mathrm{~s}^{-1}$ or less, and so remain lower than the computed synchronous rotational velocities. While the primary appears to rotate slightly slower than synchronous, the secondary may still have some way to go. However, given the weakness of the lines of the secondary, even at red wavelengths where they are enhanced, precise measurement of the latter's $v \sin i$ value is difficult, and it remains possible that the secondary is rotating synchronously.

In Figure 10, we show the observations against evolutionary tracks calculated for the exact masses we measure for HY Vir, from the Yonsei-Yale models by Demarque et al. (2004).

These models adopt a mixing length parameter $\mathrm{a}_{\mathrm{ML}}=$ 1.7431 , a solar composition of $Z_{\text {Sun }}=0.01812$, a helium enrichment law of $\Delta Y / \Delta Z=2.0$, and a mass-dependent treatment of convective core overshooting. A good match of the 


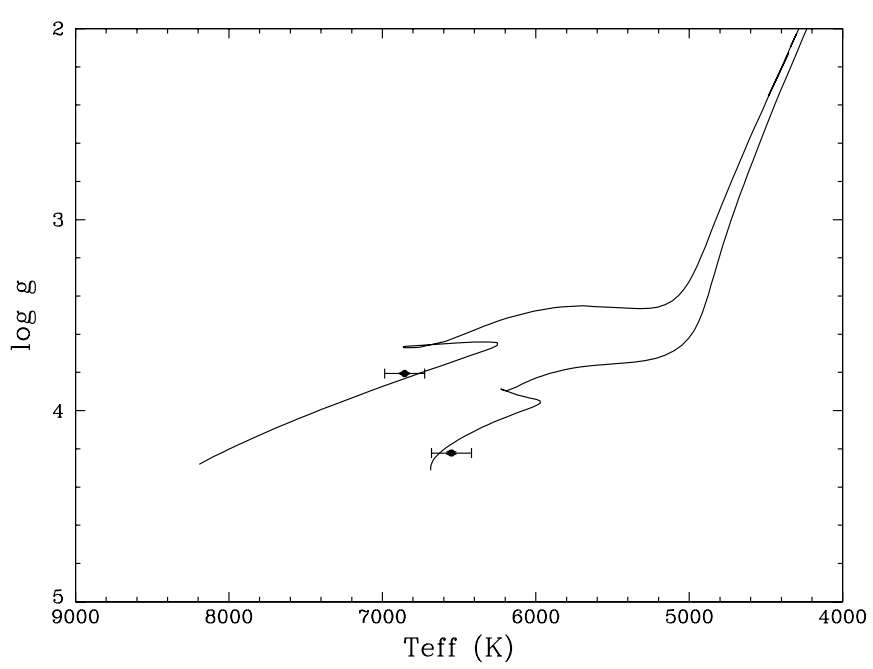

Figure 10. Observations of HY Vir and Yonsei-Yale evolutionary tracks.

models with the observations is achieved at a somewhat metalrich initial interior composition $(Z=0.027$, corresponding to $[\mathrm{Fe} / \mathrm{H}]=0.10)$, as shown in the figure. The interior composition appears to be much less metal-rich than the surface. The reason is as follows: the Am phenomenon is widely believed to be due to a surface enhancement in some heavy elements and deficit in others (Preston 1974). The current Am model assumes that the central regions of these chemically peculiar stars have a more normal composition. The central composition is what determines the evolution in the H-R diagram. The age of $1.35 \pm$ $0.10 \mathrm{Gyr}$ we infer from these models is consistent between separate fits to each star's properties. The components of HY Vir thus join those having the very best determined absolute properties.

The authors thank Bill Neely who operates and maintains the NFO WebScope for the Consortium, and who handles preliminary processing of the images and their distribution.

The authors also thank the staff at KPNO, especially Daryl Willmarth, for maintenance and preparation of the coudé-feed telescope and spectrometer.

\section{REFERENCES}

Abt, H. A., \& Morrell, N. I. 1995, ApJS, 99, 135

Alonso, A., Arribas, S., \& Martinez-Roger, C. 1996, A\&A, 313, 873

Arevalo, M. J., Lazaro, C., \& Barrera, E. 2002, IBVS No. 5241

Barden, S. C. 1985, ApJ, 295, 162

Batten, A. H., Fletcher, J. M., \& MacCarthy, D. G. 1989, Publ. Dominion Astrophys. Obs., 17, 1

Casagrande, L., Ramírez, I., Meléndez, J., et al. 2010, A\&A, 512, A54

Casas, R., \& Gomez-Forrellad, J. M. 1989, IBVS No. 3337

Claret, A. 1998, A\&AS, 131, 395

Claret, A. 2000, A\&A, 363, 1081

Claret, A., \& Hauschildt, P. H. 2003, A\&A, 412, 241

Crawford, D. L., \& Barnes, J. V. 1974, AJ, 79, 687

Demarque, P., Woo, J., Kim, Y., \& Yi, S. K. 2004, ApJS, 155, 667

Diaz-Cordoves, J., Claret, A., \& Gimenez, A. 1995, A\&AS, 110, 329

Etzel, P. B. 1981, in Photometric and Spectroscopic Binary Systems, ed. C. B. Carling \& E. Z. Kopal (Dordrecht: Reidel), 111

Fekel, F. C. 1997, PASP, 109, 514

Fekel, F. C. 2003, PASP, 115, 807

Fekel, F. C., Tomkin, J., \& Williamson, M. H. 2009, AJ, 137, 3900

Garcia-Melendo, E., Gomez-Forrellad, J. M., \& Vidal-Sainz, J. 1995, IBVS No. 4272

Grauer, A. D., Neely, A. W., \& Lacy, C. H. S. 2008, PASP, 120, 992

Hauck, B., \& Mermilliod, M. 1998, A\&AS, 129, 431

Hayes, D. S. 1978, in IAU Symp. 80, The HR Diagram, ed. A. G. D. Philip \& D. S. Hayes (Dordrecht: Reidel), 65

Huenemoerder, D. P., \& Barden, S. C. 1984, BAAS, 16, 510

Lacy, C. H., Frueh, M. L., \& Turner, A. E. 1987, AJ, 94, 1035

Lacy, C. H. S. 2011, IBVS No. 5972

Lacy, C. H. S., Torres, G., \& Claret, A. 2008, AJ, 135, 1757

Nagai, K 2008, VSB No. 46

North, P., \& Zahn, J.-P. 2004, New Astron. Rev., 48, 741

Ogloza, W., Niewiadomski, W., Barnacka, A., et al. 2008, IBVS No. 5843

Perry, C. L., \& Johnson, L. 1982, ApJS, 50, 451

Popper, D. M. 1980, ARA\&A, 18, 115

Popper, D. M., \& Etzel, P. B. 1981, AJ, 86, 102

Preston, G. W. 1974, ARA\&A, 12, 257

Renson, P., \& Manfroid, J. 2009, A\&A, 498, 961

Rodriguez, E., Sedano, J. L., Lopez de Coca, P., \& Rolland, A. 1988, IBVS No. 3174

Scarfe, C. D. 2010, Observatory, 130, 214

Selman, F. J. 2004, Proc. SPIE, 5493, 453

Southworth, J., Bruntt, H., \& Buzasi, D. L. 2007, A\&A, 467, 1215

Strassmeier, K. G., \& Fekel, F. C. 1990, A\&A, 230, 389

Stromgren, B. 1966, ARA\&A, 4, 433

Tomkin, J., \& Fekel, F. C. 2006, AJ, 131, 2652

Van Hamme, W 1993, AJ, 106, 2096

van Leeuwen, F 2007, Hipparcos, The New Reduction of the Raw Data (Astron. \& Space Sci. Library; Dordrecht: Springer) 